\title{
Serial and Macromolecular Crystallography at beamline P11, Petra III
}

\author{
Johanna Hakanpää1, Sofiane Saouane ${ }^{1}$, Guillaume Pompidor ${ }^{1}$, Eva Crosas ${ }^{1}$, Jan Meyer ${ }^{1}$, Bernhard Kistner', \\ Alessandra Henkel ${ }^{2}$, Spyros Chatziefthymiou ${ }^{1}$, Thomas A. White ${ }^{3}$, Dominik Oberthür ${ }^{3}$ \\ IPhoton Science, Deutsches Elektronen-Synchrotron DESY, Hamburg, Germany; \\ ${ }^{2}$ University of Lübeck, Lübeck, Germany; ${ }^{3}$ Center for Free-Electron Laser Science, Deutsches Elektronen-Synchrotron DESY, \\ Hamburg, Germany; \\ johanna.hakanpaa@desy.de
}

P11 at PETRA III in Hamburg is a versatile beamline for macromolecular crystallography (1). The photon energy can be adjusted between $5.5-28 \mathrm{keV}$ with the possibility of using a CdTe-detector for higher energies $(>22 \mathrm{keV})$. Beam sizes are available between $200 \times 200 \mu \mathrm{m}$ and $4 \times 9 \mu \mathrm{m}$ with a maximum photon flux of $1 \mathrm{e} 13 \mathrm{ph} / \mathrm{s}$ at $12 \mathrm{keV}$.

P11 is optimized for high-throughput crystallography. EIGER2 X 16M detector is fully integrated since spring 2021 and sample cycle of less than 2 min can be reached. The automatic sample changer at P11 is based on the unipuck format with a total capacity of 23 pucks (368 samples) and a mounting cycle of $20 \mathrm{~s}$.

Remote access was established in spring 2020 and enabled fast-track access for SARS-CoV2 related projects (e.g. 1-4) and since May 2020, almost normal user operation, despite the pandemic restrictions.

The P11 setup in the experimental hutch is very flexible and allows to accommodate various non-standard experiments e.g. via the long term proposal (LTP) scheme. Serial crystallography at P11 is enabled with sample delivery through various types of solid supports or the tape-drive setup, which also enables time-resolved experiments by the mix-and diffuse method (5). Serial data collections are implemented as fast $2 \mathrm{D}$ scans or as series of rotation wedges in the graphical user interface; full integration of tapedrive experiments is in progress. OnDA (6) is available for real time evaluation of SSX data and implementation of real-time SSX processing is in progress within an LTP.

[1] Rut et al. (2020) Nat. Chem. Biol., 2020

[2] Qiao et al. (2021) Science 10.1126/science.abf1611

[3] Oerlemans et al. (2021) RSC Medicinal Chemistry

[4] Günther et al. (2021) Science 10.1126/science.abf7945

[5] Beyerlein et al. (2017) IUCrJ 4:769

[6] Mariani et. al. (2016) J. App. Cryst. 49:1073

Keywords: serial crystallography, tape-drive, remote experiments 